\title{
Cortical Source Multivariate EEG Synchronization Analysis on Amnestic Mild Cognitive Impairment in Type 2 Diabetes
}

\author{
Dong Cui, ${ }^{1}$ Jing Liu, ${ }^{1}$ Zhijie Bian, ${ }^{2}$ Qiuli Li, ${ }^{3}$ Lei Wang, ${ }^{3}$ and Xiaoli Li ${ }^{2,4,5,6}$ \\ ${ }^{1}$ School of Information Science and Engineering, Yanshan University, Qinhuangdao 066004, China \\ ${ }^{2}$ School of Electrical Engineering, Yanshan University, Qinhuangdao 066004, China \\ ${ }^{3}$ Department of Neurology, General Hospital of Second Artillery Corps of PLA, Beijing 100875, China \\ ${ }^{4}$ State Key Laboratory of Cognitive Neuroscience and Learning and IDG/McGovern Institute for Brain Research, \\ Beijing Normal University, Beijing 100875, China \\ ${ }^{5}$ Center for Collaboration and Innovation in Brain and Learning Sciences, Beijing Normal University, Beijing 100875, China \\ ${ }^{6}$ National Key Laboratory of Cognitive Neuroscience and Learning, Beijing Normal University, Beijing 100875, China
}

Correspondence should be addressed to Xiaoli Li; xiaoli@bnu.edu.cn

Received 2 July 2014; Accepted 14 August 2014; Published 28 August 2014

Academic Editor: Jing Li

Copyright (C) 2014 Dong Cui et al. This is an open access article distributed under the Creative Commons Attribution License, which permits unrestricted use, distribution, and reproduction in any medium, provided the original work is properly cited.

\begin{abstract}
Is synchronization altered in amnestic mild cognitive impairment (aMCI) and normal cognitive functions subjects in type 2 diabetes mellitus (T2DM)? Resting eye-closed EEG data were recorded in 8 aMCI subjects and 11 age-matched controls in T2DM. Three multivariate synchronization algorithms ( $S$-estimator $(S)$, synchronization index (SI), and global synchronization index (GSI)) were used to measure the synchronization in five ROIs of sLORETA sources for seven bands. Results showed that aMCI group had lower synchronization values than control groups in parietal delta and beta 2 bands, temporal delta and beta 2 bands, and occipital theta and beta 2 bands significantly. Temporal $(r=0.629 ; P=0.004)$ and occipital $(r=0.648 ; P=0.003)$ theta $S$ values were significantly positive correlated with Boston Name Testing. In sum, each of methods reflected that the cortical source synchronization was significantly different between aMCI and control group, and these difference correlated with cognitive functions.
\end{abstract}

\section{Introduction}

The incidence and prevalence of diabetes mellitus (DM) are increasing worldwide, especially with an increase in type 2 diabetes mellitus (T2DM), which represents more than $90 \%$ of all cases of diabetes [1]. T2DM has become a significant public health problem $[2,3]$. Many recent studies indicated that T2DM was a risk factor for cognitive impairment [4, $5]$ and may cause alteration of EEG characteristics related to cognitive functions [6,7]. Mild cognitive impairment (MCI) is characterized as an early but measurable stage of cognitive impairment $[8,9]$. $\mathrm{MCI}$ is predictive of progression to dementia and the conversion rate from $\mathrm{MCI}$ to dementia was $11.65 \%$ per year [10]. One study suggests that individuals with diabetes are 1.5 times more likely to experience cognitive decline and have 1.6 times greater risk of future dementia than individuals without diabetes [11].
A promising approach to study MCI subjects is to analyze the recordings of electroencephalographic (EEG). EEG has been applied to discriminate healthy subjects with various types and severities of cognitive impairment for many years [12]. This technique is based on low cost and relatively widely available equipment in the majority of countries and is fully noninvasive. Considering the fact that MCI responds much better when treatment is commenced in the early stage of the illness than in the advanced stages, investigating the EEG signal characteristics related to MCI of diabetes patients is very important for us to understand the specific effect of brain structure and functional connective in diabetes [13].

At present, some studies analyzed the EEG recordings to study the pathophysiology of MCI in DM. Cooray et al. studied event-related potentials (ERPs) and resting EEG power spectrum in the patients with T2DM and healthy control subjects. The T2DM affected ERP with a decrease 
in N100 amplitude and an increase in P300 latency. T2DM patients had an increase in alpha-power and increase in connectivity in the alpha and theta bands across hemispheres and within lateral regions. Moreover, T2DM patients had a significant increase in connectivity in the beta band of the central region [1]. Hazari et al. studied P300 ERPs in T2DM patients without clinical evidence of central nervous system damage and nondiabetic controls. They found that P300 ERPs revealed cognitive dysfunction, which was not detected by neuropsychometric test and if the disease duration exceeds 5 years the decrease became more prominent [3].

Some studies analyzed the EEG synchronization of MCI and Alzheimer's disease (AD) but not diabetes patients. The AD patients showed lower synchronization values in beta band compared with MCI patients [14]. At group level, frontoparietal coupling of the delta and alpha rhythms progressively became abnormal through MCI and mild AD [15]. Compared with the age-matched healthy controls, AD patients had significant lower synchronization values in the beta1, beta2, and beta3 bands, and they were significantly correlated to scores of symptom severity scales [16].

An advanced method for analyzing neural activity sources (low resolution brain electromagnetic tomography (LORETA)) had been successfully applied to studying on normal and pathological aging [17]. Babiloni et al. firstly investigated the power spectrum profiles at the level of cortical EEG sources with LORETA in mild AD patients, vascular dementia ( VaD), and normal elderly people (Nold) groups and they found that alpha sources $(8-10 \mathrm{~Hz})$ were abnormal in mild AD compared with Nold and VaD subjects [18]. Then, Babiloni et al. found that the frontal white matter and the amplitude of frontal delta sources $(2-4 \mathrm{~Hz})$ were negatively correlated across MCI and AD subjects [19]; the alpha sources in parietal, occipital, and temporal areas were lower in amplitude in the AD and MCI subjects than in the Nold subjects, while the magnitude of the delta EEG sources was higher in the AD than in the Nold and MCI subjects $[20,21]$. In addition, standardized low resolution electromagnetic tomography analysis (sLORETA) allowed 3D localization of standardized current density with zero localization errors in both the time and frequency domain [22]. Caso et al. indicated that AD patients had higher theta power with scalp EEG spectral and lower alpha2 and betal values in central/temporal regions with cortical sources EEG rhythms by sLORETA than frontotemporal dementia (FTD) subjects [23]. Compared with the normal elderly subjects, the cognitive impairment subjects showed a decrease in amplitude of the alphal sources and an increase in amplitude of the delta sources [24].

However, little is known about the synchronization activity of EEG sources with amnestic MCI (aMCI) in T2DM. As far as we know, this paper is the first study about cortical source multivariate EEG synchronization analysis on aMCI in type 2 diabetes.

In the study, we recorded the resting EEG data of aMCI and nonamnesic MCI patients in T2DM. The EEG sources in time domain were estimated by sLORETA. The synchronization values of cortical EEG sources were calculated by using four kinds of multivariate synchronization methods for each subject. Then statistics analysis was done to explore the difference of the synchronization between aMCI group and control group (nonamnesic MCI patients). We also analyzed the correlation between synchronization and cognitive functions.

\section{Methods}

2.1. Subjects and Diagnostic Criteria. Nineteen T2DM patients were recruited from the Neurology Department of Second Artillery General Hospital of Beijing in China. Diagnosis and classification criteria were recommended by the World Health Organization [25]. The study was approved by Beijing Normal University ethics committee. Written informed consent was obtained from all participants before the experiment. The experiment was conducted in accordance with the Declaration of Helsinki (1964). All the patients had sufficient visual and auditory resolution to accept the neuropsychological test.

The aMCI group consisted of 8 subjects and control group consisted of 11 subjects. Inclusion criteria of the aMCI group were (1) the reporting of a decline in cognitive functioning relative to previous abilities during the past year by the patient or their families, (2) cognitive disorders as evidence by clinical assessment (with the hypomnesia of chief complaint, or in another cognitive domain, which in this study was assessed by neuropsychological test such as MMSE and MoCA, and (3) the activity of daily living being unaffected by history and evidence of independent living as assessed by Instrumental Activity of Daily Living Scale test [26].

Exclusion criteria for aMCI group were (1) MCI subjects without objective memory deficits; (2) having any other psychiatric or neurological disorders such as AD, dementia, Parkinsonism, depression, extrapyramidal disease, brain trauma, brain tumor, epilepsy, and neuropathic recession; (3) suffering from severe physical illness, such as the clinical macrovascular complications (angina, history of myocardial infarction, cerebral infarction with clinical symptoms and peripheral vascular embolism, etc.). (4) patients with severe alcoholism or drug abuse; (5) taking the sedative-hypnotic drugs and some drugs that affect the central nervous system one month before joining the group.

The control group inclusive criteria were (1) no memory impairment chief complaint; (2) activities of daily living without defect; (3) normal cognitive functions as evidenced, assessed by neuropsychological test such as MMSE and MoCA. Exclusion criteria for control group were the same as (2) -(5) items of the exclusion criteria for aMCI group.

The data including patients' name, age, gender, education years, and handedness were recorded. A series of standardized neuropsychological tests were used to assess the cognitive functions of aMCI and control groups. Each patient was evaluated by the Mini-Mental State Examination (MMSE) and Montreal Cognitive Assessment (MoCA). Attention and executive function were examined by the Wechsler Adult Intelligence Scale (WAIS) Digit Span Test [27] and the Trail Making Test parts A and B. Tests to assess memory were immediate and delayed recall measure and the delay 
recognition measure of the Rey Auditory Verbal Learning Test (AVLT) [28]. For language, semantic fluency test of the one minute verbal fluency for animals and Boston Naming Test [29] were used. The activity of daily living was tested by Instrumental Activity of Daily Living Scale (IADL) [30].

The demographic characteristics and neuropsychology of the aMCI group and the control group were investigated and statistically analyzed by independent samples $t$-test analysis with SPSS20.0. The results were shown in Table 1. The demographic data had no significant difference between the two groups. All subjects were matched regarding age, gender, and education level. Neuropsychological tests showed that the scores of MMSE and MoCA achieved a significant level $(P$ value $<0.05)$. The memory functions and language fluency were better preserved in control group than in aMCI group. The executive function and attention and daily living abilities were not significantly different between the two groups, except for the WAIS digit span test.

2.2. EEG Recording and Preprocessing. The patients were instructed to sit relaxed in a dim quiet room of Department of Neurology, General Hospital of Second Artillery Corps of PLA, Beijing, China. The room temperature was kept at $23 \pm 2^{\circ} \mathrm{C}$. The resting-state eyes-closed EEG signals were recorded using EGI system of Net Amps 300 amplifiers (Electrical Geodesics Inc. (EGI), Eugene, OR). High-density 128-channel EEG data were recorded for 5 minutes using the vertex sensor $(\mathrm{Cz})$ as the reference electrode. All electrode impedances were kept under $50 \mathrm{k} \Omega$. The signals were digitized at a rate of 1000 samples per second with a $0.10 \mathrm{~Hz} \sim$ $200 \mathrm{~Hz}$ band-pass filter. Artifacts in all channels, such as electrooculogram (EOG) and electromyogram (EMG), were eliminated visually by expert electroencephalographers (blind to the diagnosis).

2.3. Cortical Sources Analysis of the EEG Rhythms by sLORETA. Standardized low resolution electromagnetic tomographic analysis (sLORETA) is a functional imaging technique belonging to a family of standardized linear inverse solution procedures. sLORETA can model 3D distributions of the cortical sources based on extracranial measurements (i.e., scalp EEG) that would provide important information on the time course and localization of brain function [22]. sLORETA solutions are computed within a three-shell spherical head model including scalp, skull, and brain compartments. The brain compartment is restricted to the cortical gray matter/hippocampus of a head model coregistered to the Talairach probability brain atlas, which has been digitized at the Brain Imaging Center of the Montreal Neurological Institute [31]. The brain compartment includes 6239 voxels with $5 \mathrm{~mm}$ resolution. The electrode coordinates were the average location of the 10-5 system [32]. sLORETA has been successfully used in EEG and MEG analysis $[33,34]$.

In order to achieve greater confidence in the sLORETA solutions and synchronization estimates in the study, the artifact-free normalized EEG data were segmented into nonoverlapping 5-second epochs. Then the epochs of the aMCI group
TABLE 1: Demographic characteristics and neuropsychological test scores and $P$ values between aMCI and control groups in T2DM.

\begin{tabular}{lccc}
\hline Factor & aMCI group & Control group & $P$ value \\
\hline Subjects & 8 & 11 & - \\
Gender (M/F) & $5 / 3$ & $5 / 6$ & - \\
Age (years) & $70 \pm 10.784$ & $74.27 \pm 3.349$ & $\mathrm{NS}$ \\
Education level (years) & $13.88 \pm 3.441$ & $13.64 \pm 2.541$ & $\mathrm{NS}$ \\
MMSE scores & $26.88 \pm 1.885$ & $28.64 \pm 0.674$ & $0.034^{*}$ \\
MOCA scores & $22 \pm 1.852$ & $27 \pm 1.265$ & $0.000^{* *}$ \\
AVLT immediate recall & $5.06 \pm 1.664$ & $7.78 \pm 1.859$ & $0.004^{* *}$ \\
AVLT delayed recall & $3.75 \pm 3.105$ & $8.82 \pm 3.683$ & $0.005^{* *}$ \\
AVLT delay recognition & $11 \pm 3.117$ & $13.91 \pm 1.044$ & $0.034^{*}$ \\
Boston Name Test & $18.38 \pm 1.188$ & $19.82 \pm 0.405$ & $0.011^{*}$ \\
Semantic fluency & $16.88 \pm 3.314$ & $18.18 \pm 4.07$ & 0.452 \\
Trail Making test A & $68.5 \pm 19.878$ & $60.64 \pm 16.549$ & 0.36 \\
Trail Making test B & $127.5 \pm 62.551$ & $102.91 \pm 34.469$ & 0.286 \\
WAIS digit span test & $11.5 \pm 3.854$ & $14.73 \pm 2.24$ & $0.034^{*}$ \\
IADL & $1.13 \pm 1.356$ & $0.45 \pm 1.508$ & 0.333 \\
\hline
\end{tabular}

Mean values \pm standard errors of demographic characteristics and neuropsychological test scores in aMCI and control groups. $P$ values were obtained comparing aMCI and control groups with independent samples $t$-test.

aMCI, amnestic mild cognitive impairment; AVLT, the Auditory Verbal Learning Test; F, female; IADL, Instrumental Activity of Daily Living Scale; M, male; MMSE, Mini-Mental State Examination; MOCA, Montreal Cognitive Assessment; NS, not significant; WAIS, Wechsler Adult Intelligence Scale; ${ }^{*}$ indicates the $P$ value $<0.05 ;{ }^{*}{ }^{*}$ indicates the $P$ value $<0.01$.

TABLE 2: Brodmann areas into the regions of interest (ROIs).

\begin{tabular}{lc}
\hline ROI & Brodmann areas (left and right) \\
\hline Frontal & $8,9,10,11,44,45,46,47$ \\
Central & $1,2,3,4,6$ \\
Parietal & $5,7,30,39,40,43$ \\
Temporal & $20,21,22,37,38,41,42$ \\
Occipital & $17,18,19$ \\
\hline
\end{tabular}

and control group were given as an input to the original sLORETA software (http://www.uzh.ch/keyinst/loreta.htm). The amplitudes of current density as time series on 6239 voxels were calculated. For each Brodmann area, only a single voxel, which was closest to the center of the Brodmann area, was selected for the reasons that the spatial resolution of sLORETA was low; thus the single centroid voxel is an excellent representative of each Brodmann area. In addition, if the average sLORETA solutions were used, the sLORETA activity spanning a large volume might not be very meaningful. In the study, five ROIs (frontal, central, parietal, temporal, and occipital) were achieved. Each of the ROIs was made of corresponding Brodmann areas [35] (Table 2). Thus we had 58 channels' data according to these Brodmann areas.

2.4. Synchrony Measures. In this paper, we calculated the synchronization values of each $5 \mathrm{~s}$ sLORETA cortical sources epoch by using four multivariate synchronization methods. Three methods were used to measure synchronization of 
the five ROIs in the following frequency bands: delta (1.5$6 \mathrm{~Hz})$, theta $(6.5-8 \mathrm{~Hz})$, alphal $(8.5-10 \mathrm{~Hz})$, alpha2 $(10.5-$ $12 \mathrm{~Hz})$, beta1 $(12.5-18 \mathrm{~Hz})$, beta2 $(18.5-21 \mathrm{~Hz})$, beta3 $(21.5-$ $30 \mathrm{~Hz})$, gammal $(30-45 \mathrm{~Hz})$, gamma2 $(55-80 \mathrm{~Hz})$, and full $(1.5-80 \mathrm{~Hz})$ for all subjects.

2.4.1. S-Estimator. S-estimator is a kind of global synchronization index based on principal component analysis (PCA) [36]. It can measure the synchronization by measuring the eigenvalue in the correlation matrix of a multivariate set of signals and has been applied to analysis synchronous about multichannel EEG signals. The $S$-estimator is defined as

$$
S=1+\frac{\sum_{i=1}^{M} \lambda_{i}^{\prime} \log \left(\lambda_{i}^{\prime}\right)}{\log (M)},
$$

where $\lambda_{i}$ is the eigenvalue obtained from the equaltime correlation matrix $\mathbf{C}=\left[c_{i j}\right]$ [37]. In the study, $c_{i j}=$ $\left|\left\langle\exp \left(i\left(\varphi_{i}(t)-\varphi_{j}(t)\right)\right)\right\rangle_{t}\right|, i, j=1, \ldots, M$, and $\varphi_{i}(t)$ is the instantaneous phase of channel $i$ for a frequency band. $\varphi_{i}(t)$ is extracted by Gabor wavelet transform. The $\lambda_{i}^{\prime}=$ $\lambda_{i} / \sum_{i=1}^{M} \lambda_{i}$ are the normalized eigenvalues. $M$ means the channel number in the original signals. When all normalized eigenvalues are roughly of the same value, $S$ is close to zero. That is to say, the signals are statistically independent. On the contrary, if the signals are well synchronized, only a few number of eigenvalues remain prominent, and, as a result, $S$ estimator is then close to one.

2.4.2. Synchronization Index (SI). By comparing the eigenvalues of data correlation matrix $\mathbf{C}$ and surrogate correlation matrix $\mathbf{R}$, synchronization index was used to measure the synchronization of multiple neural mass populations [38]. The surrogate data were obtained with amplitude-adjusted Fourier transform (AAFT) [39] method, which randomized the phase of time series. The surrogate data not only keeps the linear properties of itself in time series but also eliminates the nonlinear correlation between multivariable time series.

The normalized SI is computed using the following equation:

$$
\mathrm{SI}_{k}= \begin{cases}\frac{\left(\lambda_{k}-\overline{\lambda_{k}^{s}}\right)}{\left(M-\overline{\lambda_{k}^{s}}\right)} & \text { if } \lambda_{k}>\left(\overline{\lambda_{k}^{s}}+P \times \mathrm{SD}_{k}\right) k=1, \ldots, M \\ 0 & \text { otherwise, }\end{cases}
$$

where $M$ represents the number of time series, and $\lambda_{k}$ represent the eigenvalues obtained from matrix $\mathbf{C}$, which is constructed as mentioned above. $\overline{\lambda_{i}^{s}}$ and $\mathrm{SD}_{k}$ denote the mean and standard deviation values of the eigenvalues of matrix R. And $P$ is a constant that determines the threshold. In the study, $P=3$ was used for a confidence probability of 0.95 . Here we focus on the largest SI and refer to it as the SI value, and SI value ranges from 0 to 1 . If SI $=0$, it means no synchronization over all the series, and high SI values can be interpreted as the presence of increased connectivity over all the series. If SI $=1$, it means that all series are complete synchronization.
2.4.3. Global Synchronization Index (GSI). Based on the description of the SI method, a new method of measuring global synchronization of multiple time series has been proposed [38]. We used the equal-time correlation method to get the correlation matrix $\mathbf{C}$, the surrogate correlation matrix $\mathbf{R}$, and their eigenvalues, like the SI method. In the GSI method, the eigenvalues of matrix $\mathbf{C}$ are normalized by dividing the eigenvalues of matrix $\mathbf{R}$ to reduce the influence of random component. The GSI is defined as

$$
\mathrm{GSI}=1+\frac{\sum_{i=1}^{M} \lambda_{i} \log \left(\lambda_{i}\right)}{\log (M)},
$$

where $\lambda_{i}$ is the normalized eigenvalues and computed as

$$
\lambda_{i}=\frac{\left(\lambda_{i} / \overline{\lambda_{i}^{s}}\right)}{\sum_{i=1}^{M} \lambda_{i} / \overline{\lambda_{i}^{s}}}, \quad i=1, \ldots, M
$$

where $\overline{\lambda_{i}^{s}}$ represent the mean values of the eigenvalues of matrix R. $M$ represents the number of time series like the SI mentioned. To understand how this estimator works, two cases should be considered. If there is no genuine correlation, the normalized eigenvalues are all equal to $1 / M$, so GSI $=0$; on the contrary, if all the time series are correlated completely, the largest normalized eigenvalue should be $M$, and the others are equal to zero; at this point, GSI $=1$.

2.5. Statistics Analysis. Independent samples $t$-test was performed in demographic tests, MMSE scores, MoCA scores, and neuropsychological tests between aMCI and control group. In the paper, the statistical analysis was used by SPSS 20.0 software (IBM SPSS, Inc., NY, Armonk, USA).

Repeated measurement of analysis of variance (ANOVA) was performed on regional synchronization values: withinsubject factors were "band" (delta, theta, alpha1, alpha2, beta1, beta2, beta3, gamma1, gamma2, and whole frequency band) and "ROIs" (frontal, central, temporal, parietal, and occipital), while between-subject factor was "group" (aMCI and control group). Age, education level, and gender were used as covariates in the statistical analysis to reduce confounding effects. Mauchly's test was used to evaluate sphericity of data; correction of the degrees of freedom was made by Greenhouse-Geisser procedure. Post hoc least significant difference (LSD) tests were used to compare group differences for each rhythm and ROIs.

Pearson's linear correlations were used between regional synchronization values and neuropsychological tests for all the subjects (aMCI and control group).

\section{Results}

3.1. Statistical Comparisons of the Synchronization Values. Figure 1 showed the bar graphs of regional synchronization values which were calculated by the S-estimator, SI and GSI, and the results of statistical analysis. The three synchronization measures of aMCI group decreased in all bands relative to a statistical interaction among the factors, which were the groups (aMCI and control group), bands (delta, theta, alpha1, 
alpha2, beta1, beta2, and beta3), and ROIs (frontal, central, temporal, parietal, and occipital). The figure did not show gammal, gamma2, and the whole frequency band because synchronous values in these bands were not significantly different between two groups.

In Figure 1, frontal S, SI, and GSI values of aMCI group were not significantly different. The three synchronization values in parietal and temporal regions all were significantly different in delta and beta 2 bands between aMCI and control group. The figure also showed that occipital theta and beta 2 synchronization values measured by the three methods were more significantly different between the two groups, especially in the beta2 band.

Performing the repeated measurement of ANOVA on $S$ values, the group effect $(F(1,19)=15.912, P<0.001)$, interaction Band $*$ ROI effect $(F(36,36)=13.442, P<0.001)$ and interaction Band $*$ Group effect $(F(9,162)=7.844, P<$ $0.001)$ were significant. The interaction Band $* \mathrm{ROI} *$ Group was not significant $(F(36,648)=1.253, P=0.223)$. For SI values, the group effect $(F(1,19)=16.046, P<$ $0.001)$, interaction Band $*$ ROI effect $(F(36,36)=4.324$, $P<0.001)$, interaction Band $*$ Group effect $(F(9,162)=$ 7.630, $P<0.001)$ and interaction Band $*$ ROI $*$ Group effect $(F(36,648)=1.662, P=0.035)$ were significant. And for the GSI values, the group effect $(F(1,19)=15.617, P<0.001)$, interaction Band $*$ ROI effect $(F(36,36)=15.739, P<0.001)$, interaction Band $*$ Group effect $(F(9,162)=5.091, P<$ $0.001)$ were significant. The interaction Band $* \mathrm{ROI} *$ Group was not significant $(F(36,648)=1.085, P=0.363)$.

3.2. Correlation Analysis. In order to investigate the relationships between cognitive functions and the synchronization values that are computed by the $S$-estimator, SI, and GSI method respectively, Pearson's correlation analysis was performed between the synchronization values (different bands and ROIs) and the scores of neuropsychological tests (MoCA, AVLT immediate recall, AVLT delay recall, AVLT delay recognition, WAIS digit span test, semantic fluency, Boston Name Testing, Trail Making test A, and Trail Making test B) in all subjects as a whole group. Based on Bonferroni method, the significant correlation values $r$ and $P$ values $(P<0.01)$ were corrected. We found that only temporal theta $S$ values ( $r=0.629, P=0.004)$, occipital theta $S$ values $(r=0.648$, $P=0.003$ ), and Boston Name Testing were strict correlated significantly. Scatter grams were illustrated in Figure 2.

\section{Discussion}

In this paper, we studied the synchronizations of resting cortical sources with multichannel synchronization algorithms to analyze the synchronization mechanism of the amnestic MCI subjects in T2DM. To our knowledge, we firstly applied the source EEG synchronization with multichannel method in amnestic MCI with T2DM.

Our results showed that the synchronization values estimated by $S$-estimator, SI, and GSI methods decreased significantly in beta band and were similar to those of MCI and $\mathrm{AD}$ but not diabetes patients [40]. The synchronization

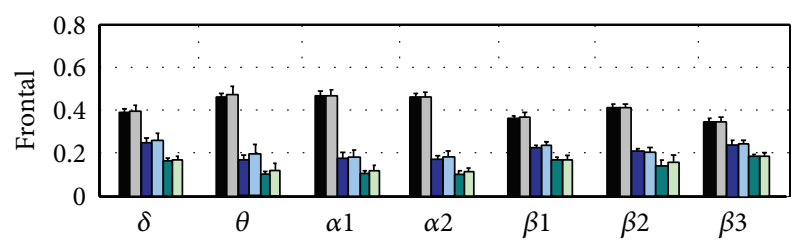

(a)

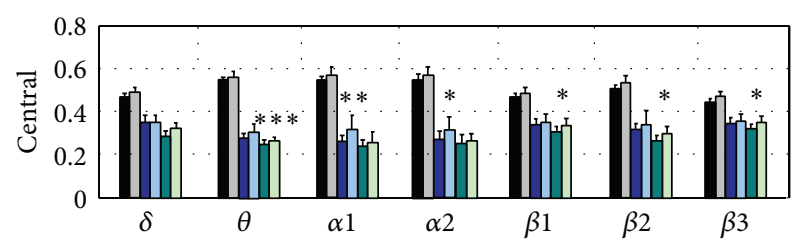

(b)

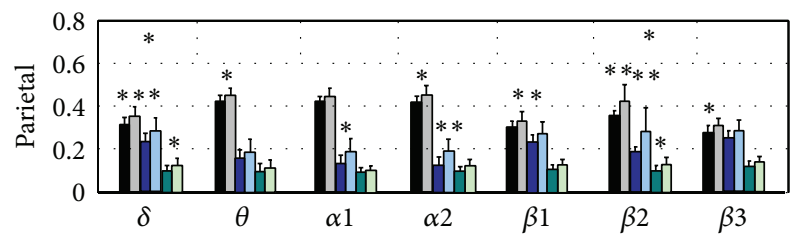

(c)

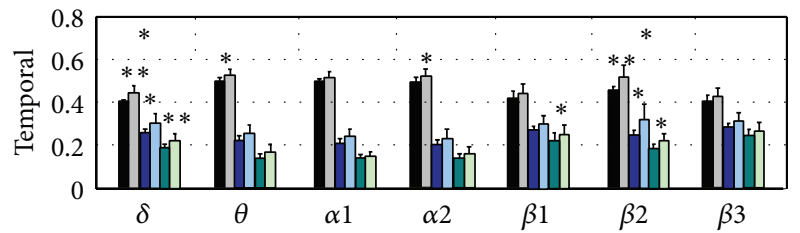

(d)

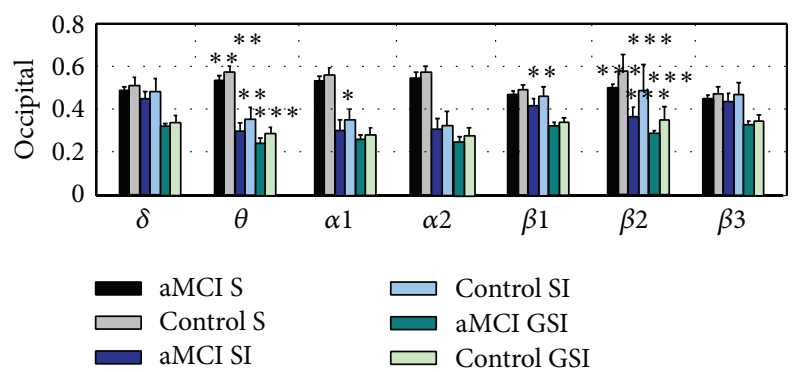

(e)

FIGURE 1: Regional synchronization values (mean \pm standard error) of delta, theta, alpha1, alpha2, beta1, beta2, and beta3 bands which were calculated by the $S$-estimator, SI, and GSI method in aMCI and control group. Post hoc analysis results were graphically displayed inside the horizontal bar over the graph: asterisks were referred to aMCI/control comparison: one asterisk means $P<0.05$, two mean $P<0.01$, and three mean $P<0.001$.

values also decreased significantly in delta and theta bands of aMCI group in T2DM; it may be due to the source EEG synchronization or due to the fact that the patients were T2DM. We also found that in aMCI groups the parietal, temporal, and occipital networks show reduced synchronization observably in these three methods, while central networks reduced observably except in $S$-estimator. The synchronization values in frontal area reduced but all of them have no significance. 


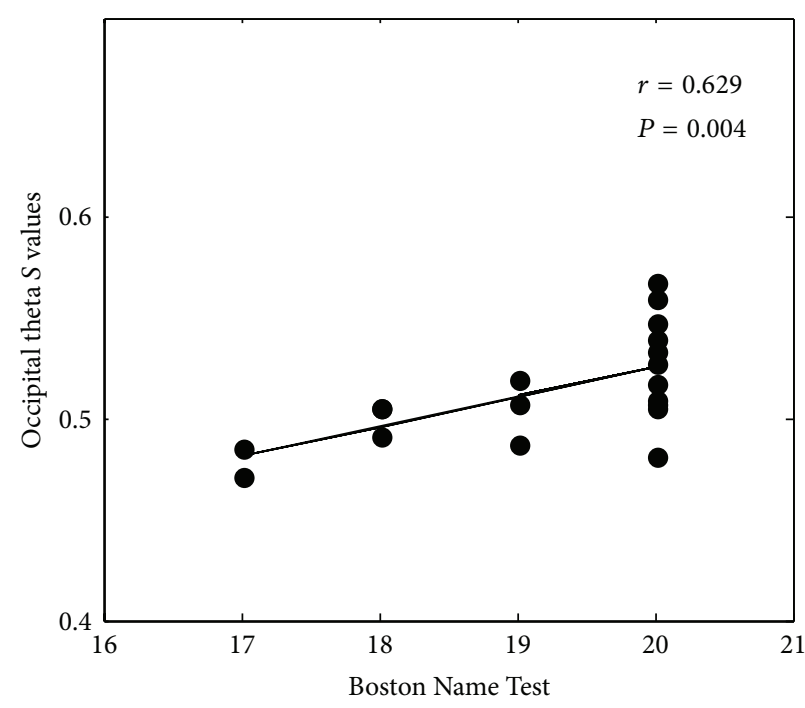

(a)

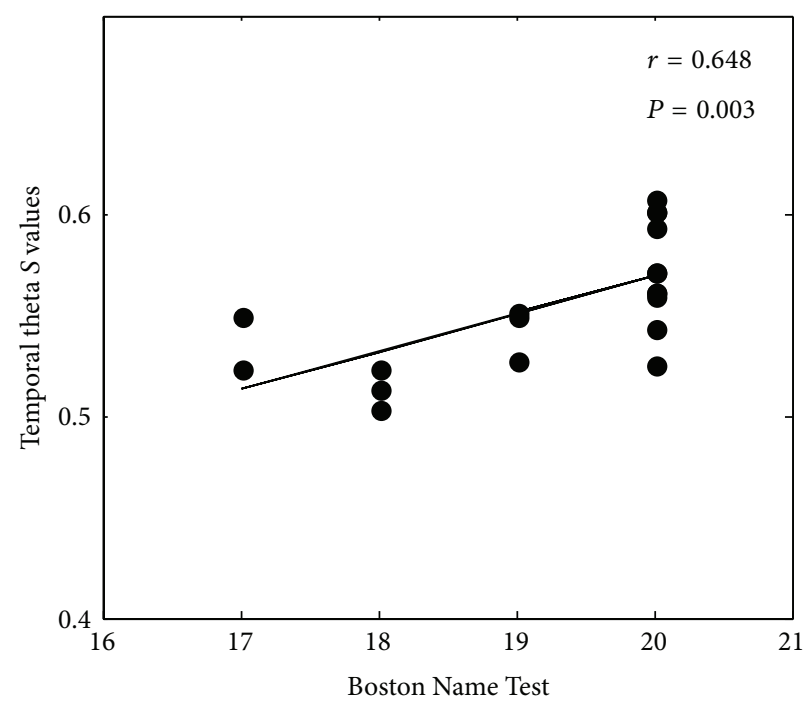

(b)

FIGURE 2: Correlation analysis between cortical source EEG synchronous and the scores of the neuropsychological tests in all subjects as a whole group (only significant correlations were displayed). $S$ values as a function of Boston Name Test within temporal theta and occipital theta were reported; the correlation was $r=0.629, P=0.004$ and $r=0.648, P=0.003$, respectively.

Recent studies in MCI and AD but not diabetes patients showed that cortical sources of EEG rhythms were abnormal in temporal [23, 41], frontal, parietal, central, and occipital regions $[35,42]$, and the synchronization shows different in parietal, frontal [43, 44], temporal, and occipital regions [45].

In these three methods, each measure reflected different aspects of change in brain synchronization during amnestic mild cognitive impairment in T2DM. We also found that these methods have something in common; the results of three methods showed that aMCI group has lower synchronization values than control groups basically in all bands and ROIs, especially in parietal, temporal, and occipital regions. In these three methods, the occipital beta2 synchronization values were significantly lower in aMCI group than in control group.

At present, several studies have successfully analyzed the synchronization characteristics between multiple neural signals in MCI and AD patients but not diabetes patients. Pijnenburg et al. investigated the behavior of synchronization likelihood (SL) of multichannel EEG in AD, MCI, and cognitively healthy controls, both at rest and during a working memory task. Decrease of beta $(12-30 \mathrm{~Hz})$ band synchronization occurs in mild $\mathrm{AD}$, both in a resting condition and during a working memory task [40]. Direction of information flux within EEG functional coupling at electrode pairs was performed by directed transfer function (DTF) in normal elderly, amnesic MCI, and mild AD subjects at rest condition (closed eyes). Parietal to frontal direction of the information flux within EEG functional coupling was stronger in normal elderly than in MCI and/or AD subjects, namely, alpha and beta rhythms. In contrast, the directional flow within interhemispheric EEG functional coupling did not discriminate among the three groups. These results suggest that directionality of parietal-to-frontal EEG synchronization is abnormal not only in $\mathrm{AD}$ but also in amnesic MCI [43]. Lee et al. analyzed the EEG of AD patients by using the global synchronization index, which is the same as SI method in this paper. They found that the GSI values in the betal $(13-18 \mathrm{~Hz})$, beta2 $(19-21 \mathrm{~Hz})$, beta3 $(22-30 \mathrm{~Hz})$, and gamma $(30-50 \mathrm{~Hz})$ bands were significantly lower in $\mathrm{AD}$ patients than in normal control. GSI values in the beta and gamma bands were positively correlated with the MMSE scores in all participants (AD and normal control) [16]. These studies indicated abnormal strength and direction of synchronization in MCI and AD patients.

The results of cortical sources analysis showed that the delta cortical sources in the whole brain, alpha cortical sources in occipital areas [46], and theta cortical sources in parietal and occipital were inordinate obviously in MCI and $\mathrm{AD}$ but not diabetes patients $[35,41]$. However, surface topography provides limited information about source brain structures causing the changes in EEG synchronization. The synchronizations of cortical sources may be more accurate than the synchronizations of scalp EEG. Knyazeva et al. used local autoregressive average (LAURA) method to generate the sources of scalp EEG in AD patients and age-matched controls one-year follow-up. The main effect of AD was hyposynchronization in the medial temporal and frontal regions and relative hypersynchronization in posterior cingulate, precuneus, cuneus, and parietotemporal cortices. Rapidly progressing $\mathrm{AD}$ patients showed a significant reduction in synchronization with time, manifest in left frontotemporal cortex. Thus, the evolution of source EEG synchronization over time is correlated with the rate of disease progression and should be considered as a cost-effective AD biomarker [44].

We tried to figure out some statistically significant correlation between source of EEG synchronization and cognitive 
functions of patients revealed by neuropsychological test. The $S$, SI, and GSI values of theta or beta correlated positively with the Boston Name Testing, and the $S$, SI, and GSI values of delta or beta correlated positively with WAIS digit span test. Delta and beta GSI values and alpha SI values positively correlated with MoCA scores. Based on Bonferroni method, only temporal theta $S$ values $(r=0.629, P=0.004)$, occipital theta $S$ values $(r=0.648, P=0.003)$, and Boston Name Testing were strict correlated significantly.

Several studies used coherence as a measure of interdependencies between EEG or MEG data and found a significant loss of alpha band coherence in $\mathrm{AD}[47,48]$. Babiloni et al. pointed out that the intensity of alpha value changes in pathological aging as a function of the global cognitive level [49]. The beta band has classically been related to excitatory activity and cognitive processes that deteriorate during $\mathrm{AD}$ [14]. Furthermore, beta spectral power has also been shown to decrease in $\mathrm{AD}$ and the decrease of beta band synchronization is related to diminished semantic memory function [50]. Koenig et al. found that the delta band synchronization increased almost continuously with increasing cognitive impairments [14], while Jelic et al. reported that activity in $\mathrm{AD}$ patients reduces in delta and theta [51]. However, there are still controversial findings concerning coherence in the delta and theta bands, with some studies finding a decreased coherence and others finding an increased coherence [52].

The mechanisms of cognitive impairment in diabetes remain to be elucidated. Chronic hyperglycemia was one of the determinants of cognitive decline in people with T2DM [53]. Hyperglycemia may be directly toxic to the neuron, leading to its degeneration [54]. The deleterious effects of hyperglycemia were mediated through an increased influx of glucose through the polyol pathway forming sorbitol and fructose, oxidative stress, and nonenzymatic glycation of biomolecules resulting in advanced glycation end products (AGE) [53]. Hypoglycemia and hemodynamic changes in persons with T2DM are also associated with cognitive impairment, including lacunae and microinfarcts [55]. A few studies showed that patients with T2DM have increased arterial stiffness [56] associated with cognitive impairment [57]. Long duration of DM may increase the risk of cognitive dysfunction, causing atherosclerosis, stroke, and cerebral infarctions [58]. Studies have suggested that duration of diabetes, use of glucose-lowering medications, and degree of glucose control may modulate the risk of cognitive impairment in older persons with T2DM [59]. Insulin resistance contributes through the indirect mechanism of upregulating hypothalamic-pituitary-adrenal axis, thereby causing hypercortisolemia related cognitive dysfunction [60]. Hypertension usually exists as a comorbid condition with DM and may be a part of a larger metabolic syndrome, including hyperglycemia, hyperinsulinemia, and dyslipidemia. Hypertension and diabetes, when combined, increase the risk of cognitive impairment [61]. Other likely mechanisms of cognitive dysfunction in T2DM were extensive leukoaraiosis (white matter hyperintense lesions, WMHLs), atrophy in the region of hippocampus, and amygdala [62]. Reduced volumes of the amygdala and the hippocampus in T2DM patients may underlie deficits associated with learning and memory [55]. A report on MRI abnormalities and cognitive changes found substantial white matter lesions and subcortical atrophies in T2DM patients.

Our study had several limitations. First, our sample was relatively small, only 19 subjects in T2DM. Making additional studies with larger samples is necessary to generalize our conclusions. Second, there were no normal elderly subjects or $\mathrm{AD}$ patients included. The comparison of the synchronization values was independent only in amnestic MCI subjects and normal cognitive function subjects in T2DM. Adding the two groups to contrast analysis, the conclusion should be enriched and the results should be more convincing. Furthermore, the EEG signals were not analyzed when the patients were performing cognitive tasks. Recording data of more subjects, including normal elderly subjects, T2DM, and $\mathrm{AD}$ patients, and study of synchronization both at rest and performing cognitive tasks are still needed in further research.

\section{Conclusion}

In this paper, we analyzed the source EEG synchronization in amnesic MCI and normal cognitive function subjects in T2DM by three synchrony measures ( $S$-estimator, SI, and GSI) and compared these values to explore the difference between the two groups. Results showed that cortical EEG source synchronization values illustrated significant difference between aMCI and control group. The aMCI group had significantly lower parietal and temporal S, SI, and GSI values in delta and beta2 bands. Occipital theta and beta2 synchronization values measured by the three methods were more significantly different between the two groups, especially in the beta 2 band. Temporal and occipital theta $S$ values were significantly positively correlated with Boston Name Testing. In sum, the synchronization of cortical EEG source was different between the amnestic and normal cognitive function subjects in T2DM and the synchronization was correlated with some cognitive functions.

\section{Conflict of Interests}

The authors declare that there is no conflict of interests regarding the publication of this paper.

\section{Acknowledgments}

This work was supported by National Natural Science Funds for Distinguished Young Scholar of China (61025019); National Natural Science Foundation of China (61102005); and Natural Science Foundation of Hebei Province of China (F2014203132).

\section{References}

[1] G. Cooray, E. Nilsson, Å. Wahlin, E. J. Laukka, K. Brismar, and T. Brismar, "Effects of intensified metabolic control on CNS 
function in type 2 diabetes," Psychoneuroendocrinology, vol. 36, no. 1, pp. 77-86, 2011.

[2] P. Zimmet, K. G. M. M. Alberti, and J. Shaw, "Global and societal implications of the diabetes epidemic," Nature, vol. 414, no. 6865, pp. 782-787, 2001.

[3] M. A. H. Hazari, B. Ram Reddy, N. Uzma, and B. S. Kumar, "Cognitive impairment in type 2 diabetes mellitus," International Journal of Diabetes Mellitus, 2011.

[4] K. Yaffe, K. Lindquist, A. V. Schwartz et al., "Advanced glycation end product level, diabetes, and accelerated cognitive aging," Neurology, vol. 77, no. 14, pp. 1351-1356, 2011.

[5] G. Cheng, C. Huang, H. Deng, and H. Wang, "Diabetes as a risk factor for dementia and mild cognitive impairment: a metaanalysis of longitudinal studies," Internal Medicine Journal, vol. 42, no. 5, pp. 484-491, 2012.

[6] C. Messier, "Impact of impaired glucose tolerance and type 2 diabetes on cognitive aging," Neurobiology of Aging, vol. 26, supplement 1, pp. S26-S30, 2005.

[7] C. T. Kodl and E. R. Seaquist, "Cognitive dysfunction and diabetes mellitus," Endocrine Reviews, vol. 29, no. 4, pp. 494-511, 2008.

[8] A. Pan, M. Lucas, Q. Sun et al., "Bidirectional association between depression and type 2 diabetes mellitus in women," Archives of Internal Medicine, vol. 170, no. 21, pp. 1884-1891, 2010.

[9] P. S. Koekkoek, G. E. H. M. Rutten, C. Ruis et al., "Mild depressive symptoms do not influence cognitive functioning in patients with type 2 diabetes," Psychoneuroendocrinology, vol. 38, no. 3, pp. 376-386, 2013.

[10] L. Li, Y. Wang, J. Yan et al., "Clinical predictors of cognitive decline in patients with mild cognitive impairment: the Chongqing aging study," Journal of Neurology, vol. 259, no. 7, pp. 1303-1311, 2012.

[11] T. Cukierman, H. C. Gerstein, and J. D. Williamson, "Cognitive decline and dementia in diabetes-systematic overview of prospective observational studies," Diabetologia, vol. 48 , no. 12 , pp. 2460-2469, 2005.

[12] J. Breslau, A. Starr, N. Sicotte, J. Higa, and M. S. Buchsbaum, "Topographic EEG changes with normal aging and SDAT," Electroencephalography and Clinical Neurophysiology, vol. 72, no. 4, pp. 281-289, 1989.

[13] S. A. Ebady, M. A. Arami, and M. H. Shafigh, "Investigation on the relationship between diabetes mellitus type 2 and cognitive impairment," Diabetes Research and Clinical Practice, vol. 82, no. 3, pp. 305-309, 2008.

[14] T. Koenig, L. Prichep, T. Dierks et al., "Decreased EEG synchronization in Alzheimer's disease and mild cognitive impairment," Neurobiology of Aging, vol. 26, no. 2, pp. 165-171, 2005.

[15] C. Babiloni, R. Ferri, G. Binetti et al., "Fronto-parietal coupling of brain rhythms in mild cognitive impairment: a multicentric EEG study," Brain Research Bulletin, vol. 69, no. 1, pp. 63-73, 2006.

[16] S. H. Lee, Y. M. Park, D. W. Kim, and C. H. Im, "Global synchronization index as a biological correlate of cognitive decline in Alzheimer's disease," Neuroscience Research, vol. 66, no. 4, pp. 333-339, 2010.

[17] C. Babiloni, G. Binetti, E. Cassetta et al., "Sources of cortical rhythms change as a function of cognitive impairment in pathological aging: a multicenter study," Clinical Neurophysiology, vol. 117, no. 2, pp. 252-268, 2006.
[18] C. Babiloni, G. Binetti, E. Cassetta et al., "Mapping distributed sources of cortical rhythms in mild Alzheimer's disease: a multicentric EEG study," NeuroImage, vol. 22, no. 1, pp. 57-67, 2004.

[19] C. Babiloni, G. Frisoni, M. Steriade et al., "Frontal white matter volume and delta EEG sources negatively correlate in awake subjects with mild cognitive impairment and Alzheimer's disease," Clinical Neurophysiology, vol. 117, no. 5, pp. 1113-1129, 2006.

[20] C. Babiloni, G. B. Frisoni, M. Pievani et al., "White matter vascular lesions are related to parietal-to-frontal coupling of EEG rhythms in mild cognitive impairment," Human Brain Mapping, vol. 29, no. 12, pp. 1355-1367, 2008.

[21] C. Babiloni, G. B. Frisoni, M. Pievani et al., "Hippocampal volume and cortical sources of EEG alpha rhythms in mild cognitive impairment and Alzheimer disease," NeuroImage, vol. 44, no. 1, pp. 123-135, 2009.

[22] R. D. Pascual-Marqui, "Standardized low-resolution brain electromagnetic tomography (sLORETA): technical details," Methods and Findings in Experimental and Clinical Pharmacology, vol. 24, pp. 5-12, 2002.

[23] F. Caso, M. Cursi, G. Magnani et al., "Quantitative EEG and LORETA: valuable tools in discerning FTD from AD?" Neurobiology of Aging, vol. 33, no. 10, pp. 2343-2356, 2012.

[24] C. Babiloni, C. del Percio, R. Lizio et al., "Cortical sources of resting state electroencephalographic alpha rhythms deteriorate across time in subjects with amnesic mild cognitive impairment," Neurobiology of Aging, vol. 34, no. 1, pp. 130-142, 2014.

[25] G. Puavilai, S. Chanprasertyotin, and A. Sriphrapradaeng, "Diagnostic criteria for diabetes mellitus and other categories of glucose intolerance: 1997 criteria by the Expert Committee on the Diagnosis and Classification of Diabetes Mellitus (ADA), 1998 WHO consultation criteria, and 1985 WHO criteria," Diabetes Research and Clinical Practice, vol. 44, no. 1, pp. 21-26, 1999.

[26] R. C. Petersen, "Mild cognitive impairment as a diagnostic entity," Journal of Internal Medicine, vol. 256, no. 3, pp. 183-194, 2004.

[27] A. Orsini, D. Grossi, E. Capitani, M. Laiacona, C. Papagno, and G. Vallar, "Verbal and spatial immediate memory span: normative data from 1355 adults and 1112 children," The Italian Journal of Neurological Sciences, vol. 8, no. 6, pp. 539-548, 1987.

[28] A. Rey, "Memorisation d'une serie de 15 mots en 5 repetitions," in Lexamen clinique en psychologie, Universitaires de France Press, Paris, France, 1958.

[29] G. Novelli, "Three clinical tests for the assessment of lexical retrieval and production. Norms from 320 normal subjects," Archivio di Psicologia, Neurologia e Psichiatria, vol. 47, pp. 477506, 1986.

[30] M. P. Lawton and E. M. Brody, "Assessment of older people: self-maintaining and instrumental activities of daily living," The Gerontologist, vol. 9, no. 3, pp. 179-186, 1969.

[31] J. Talairach and P. Tournoux, Co-Planar Stereotaxic Atlas of the Human Brain, Georg Thieme, Stuttgart, Germany, 1988.

[32] V. Jurcak, M. Okamoto, A. Singh, and I. Dan, "Virtual 1020 measurement on MR images for inter-modal linking of transcranial and tomographic neuroimaging methods," Neurolmage, vol. 26, no. 4, pp. 1184-1192, 2005.

[33] K. Sekihara, M. Sahani, and S. S. Nagarajan, "Localization bias and spatial resolution of adaptive and non-adaptive spatial filters for MEG source reconstruction," NeuroImage, vol. 25, no. 4, pp. 1056-1067, 2005. 
[34] Y. Du, J. X. Zhang, Z. Xiao, and R. Wu, "Neural systems and time course of proactive interference in working memory," Annual International Conference of the IEEE Engineering in Medicine and Biology Society, vol. 2007, pp. 1136-1139, 2007.

[35] C. Babiloni, P. J. Visser, G. Frisoni et al., "Cortical sources of resting EEG rhythms in mild cognitive impairment and subjective memory complaint," Neurobiology of Aging, vol. 31, no. 10, pp. 1787-1798, 2010.

[36] C. Carmeli, M. G. Knyazeva, G. M. Innocenti, and O. De Feo, "Assessment of EEG synchronization based on state-space analysis," NeuroImage, vol. 25, no. 2, pp. 339-354, 2005.

[37] P. Šeba, "Random matrix analysis of human EEG data," Physical Review Letters, vol. 91, no. 19, Article ID 198104, 2003.

[38] D. Cui, X. Liu, Y. Wan, and X. Li, "Estimation of genuine and random synchronization in multivariate neural series," Neural Networks, vol. 23, no. 6, pp. 698-704, 2010.

[39] T. Schreiber and A. Schmitz, "Improved surrogate data for nonlinearity tests," Physical Review Letters, vol. 77, no. 4, pp. 635-638, 1996.

[40] Y. A. L. Pijnenburg, Y. Vd Made, A. M. van Cappellen van Walsum, D. L. Knol, P. Scheltens, and C. J. Stam, "EEG synchronization likelihood in mild cognitive impairment and Alzheimer's disease during a working memory task," Clinical Neurophysiology, vol. 115, no. 6, pp. 1332-1339, 2004.

[41] F. Vecchio, L. Valeriani, P. Buffo et al., "Cortical sources of EEG rhythms in congestive heart failure and Alzheimer's disease," International Journal of Psychophysiology, vol. 86, no. 1, pp. 98107, 2012.

[42] P. M. Rossini, C. del Percio, P. Pasqualetti et al., "Conversion from mild cognitive impairment to Alzheimer's disease is predicted by sources and coherence of brain electroencephalography rhythms," Neuroscience, vol. 143, no. 3, pp. 793-803, 2006.

[43] C. Babiloni, R. Ferri, G. Binetti et al., "Directionality of EEG synchronization in Alzheimer's disease subjects," Neurobiology of Aging, vol. 30, no. 1, pp. 93-102, 2009.

[44] M. G. Knyazeva, C. Carmeli, A. Khadivi, J. Ghika, R. Meuli, and R. S. Frackowiak, "Evolution of source EEG synchronization in early Alzheimer's disease," Neurobiology of Aging, vol. 34, no. 3, pp. 694-705, 2013.

[45] M. G. Knyazeva, M. Jalili, A. Brioschi et al., "Topography of EEG multivariate phase synchronization in early Alzheimer's disease," Neurobiology of Aging, vol. 31, no. 7, pp. 1132-1144, 2010.

[46] C. Babiloni, G. B. Frisoni, M. Pievani et al., "White-matter vascular lesions correlate with alpha EEG sources in mild cognitive impairment," Neuropsychologia, vol. 46, no. 6, pp. 1707-1720, 2008.

[47] J. V. Haxby, C. L. Grady, R. Duara, N. Schlageter, G. Berg, and S. I. Rapoport, "Neocortical metabolic abnormalities precede nonmemory cognitive defects in early Alzheimer's-type dementia," Archives of Neurology, vol. 43, no. 9, pp. 882-885, 1986.

[48] D. P. Holschneider and A. F. Leuchter, "Activity in aging and dementia," Brain Topography, vol. 8, no. 2, pp. 169-180, 1995.

[49] C. Babiloni, G. Binetti, A. Cassarino et al., "Sources of cortical rhythms in adults during physiological aging: a multicentric EEG study," Human Brain Mapping, vol. 27, no. 2, pp. 162-172, 2006.

[50] A. C. Papanicolaou, D. W. Loring, G. Deutsch, and H. M. Eisenberg, "Task-related EEG asymmetries: a comparison of alpha blocking and beta enhancement.," The International Journal of Neuroscience, vol. 30, no. 1-2, pp. 81-85, 1986.
[51] V. Jelic, T. Dierks, K. Amberla, O. Almkvist, B. Winblad, and A. Nordberg, "Longitudinal changes in quantitative EEG during long-term tacrine treatment of patients with Alzheimer's disease," Neuroscience Letters, vol. 254, no. 2, pp. 85-88, 1998.

[52] T. Locatelli, M. Cursi, D. Liberati, M. Franceschi, and G. Comi, "EEG coherence in Alzheimer's disease," Electroencephalography and Clinical Neurophysiology, vol. 106, no. 3, pp. 229-237, 1998.

[53] A. M. A. Brands, R. P. C. Kessels, E. H. F. De Haan, L. J. Kappelle, and G. J. Biessels, "Cerebral dysfunction in type 1 diabetes: effects of insulin, vascular risk factors and blood-glucose levels," European Journal of Pharmacology, vol. 490, no. 1-3, pp. 159168, 2004.

[54] J. P. Klein and S. G. Waxman, "The brain in diabetes: molecular changes in neurons and their implications for end-organ damage," The Lancet Neurology, vol. 2, no. 9, pp. 548-554, 2003.

[55] E. S. C. Korf, L. R. White, P. H. Scheltens, and L. J. Launer, "Brain aging in very old men with type 2 diabetes: the Honolulu-Asia Aging Study," Diabetes Care, vol. 29, no. 10, pp. 2268-2274, 2006.

[56] M. Zhang, Y. Bai, P. Ye et al., "Type 2 diabetes is associated with increased pulse wave velocity measured at different sites of the arterial system but not augmentation index in a Chinese population," Clinical Cardiology, vol. 34, no. 10, pp. 622-627, 2011.

[57] S. Mehrabian, M. Raycheva, A. Gateva et al., "Cognitive dysfunction profile and arterial stiffness in type 2 diabetes," Journal of the Neurological Sciences, vol. 322, no. 1-2, pp. 152-156, 2012.

[58] D. G. Bruce, W. A. Davis, G. P. Casey et al., "Predictors of cognitive decline in older individuals with diabetes," Diabetes Care, vol. 31, no. 11, pp. 2103-2107, 2008.

[59] M. Munshi, L. Grande, M. Hayes et al., "Cognitive dysfunction is associated with poor diabetes control in older adults," Diabetes Care, vol. 29, no. 8, pp. 1794-1799, 2006.

[60] Z. S. K. Lee, J. C. N. Chan, V. T. F. Yeung et al., "Plasma insulin, growth hormone, cortisol, and central obesity among young Chinese type 2 diabetic patients," Diabetes Care, vol. 22, no. 9, pp. 1450-1457, 1999.

[61] L. B. Hassing, S. M. Hofer, S. E. Nilsson et al., "Comorbid type 2 diabetes mellitus and hypertension exacerbates cognitive decline: evidence from a longitudinal study," Age and Ageing, vol. 33, no. 4, pp. 355-361, 2004.

[62] T. den Heijer, S. E. Vermeer, E. J. van Dijk et al., “Type 2 diabetes and atrophy of medial temporal lobe structures on brain MRI," Diabetologia, vol. 46, no. 12, pp. 1604-1610, 2003. 

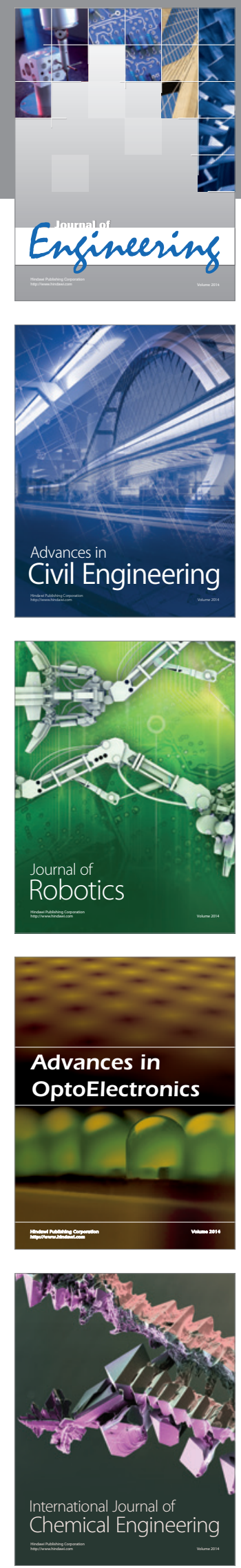

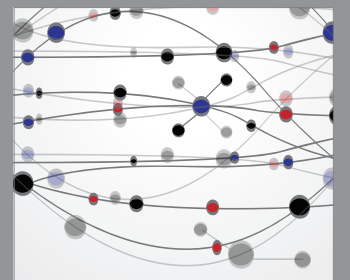

The Scientific World Journal
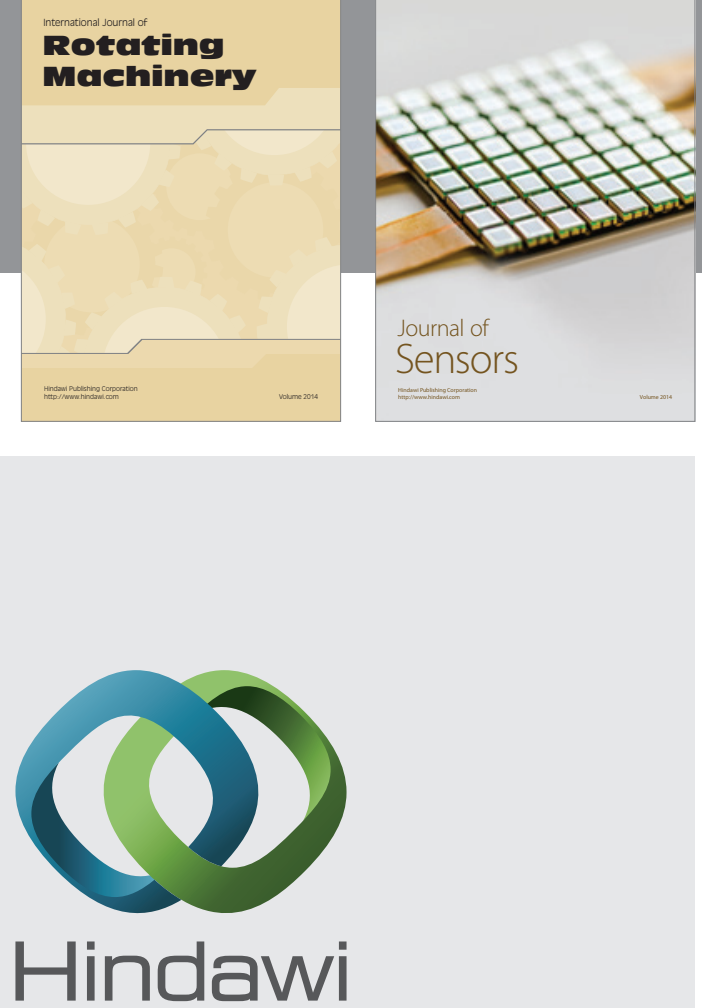

Submit your manuscripts at http://www.hindawi.com
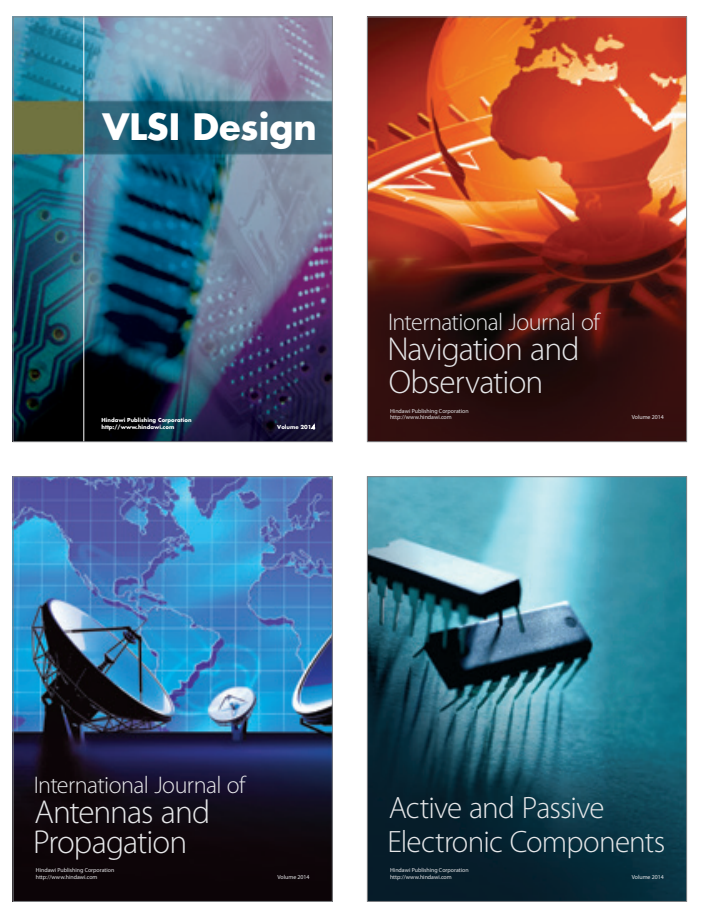
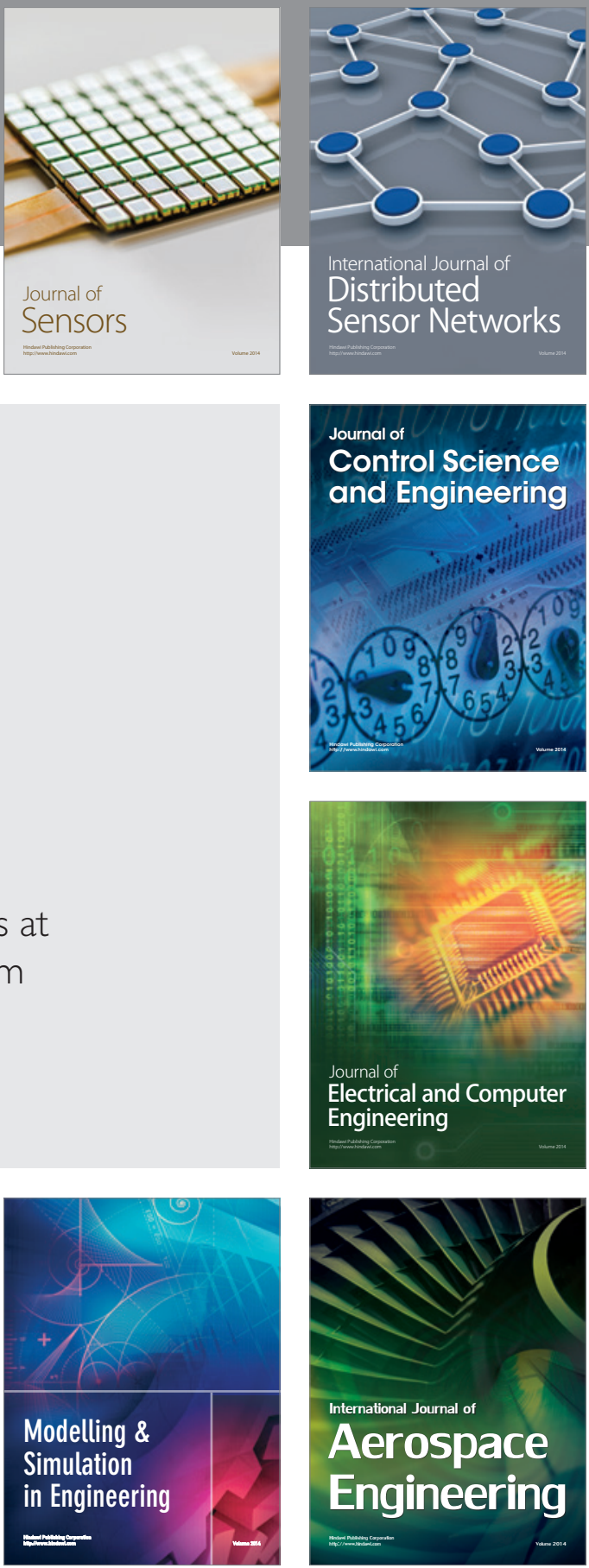

Journal of

Control Science

and Engineering
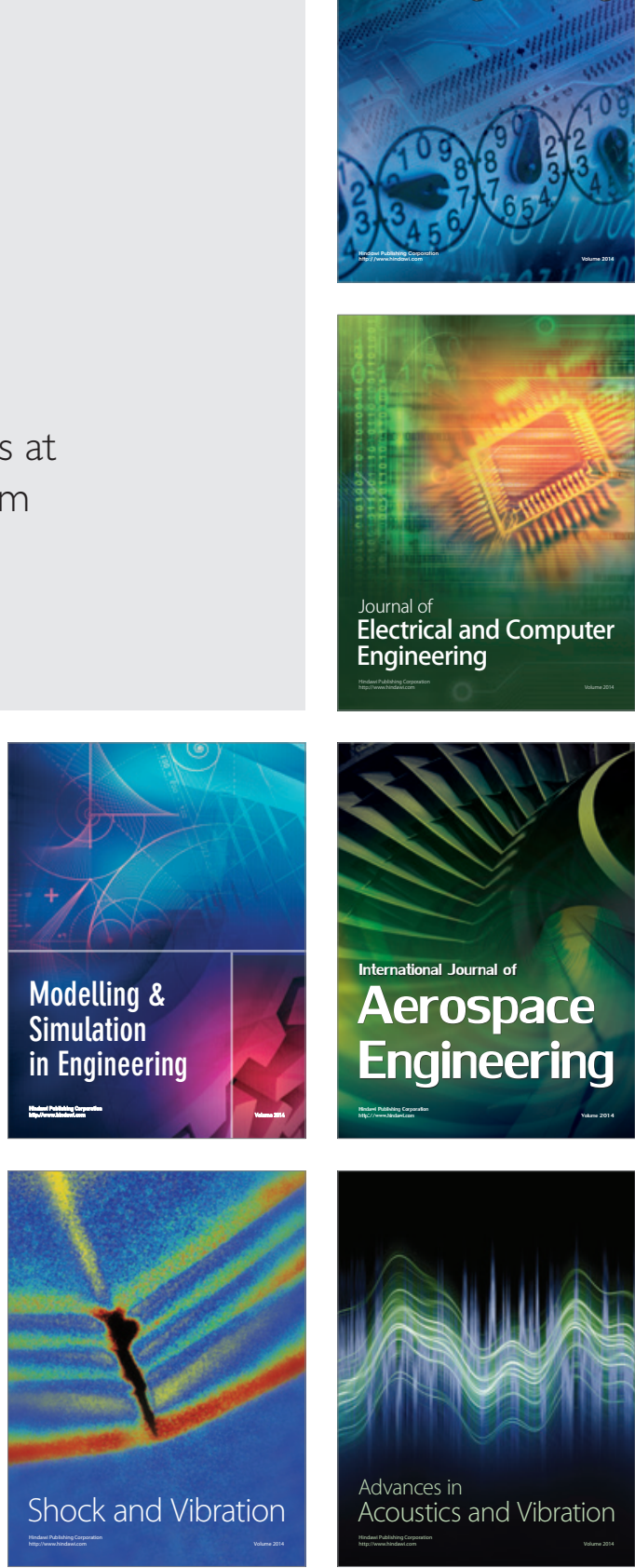